\title{
Mesenchymal stromal cell conditioned media for lung disease: a systematic review and meta-analysis of preclinical studies
}

\author{
Chimobi Emukah', Evan Dittmar', Rija Naqvi ${ }^{1}$, John Martinez ${ }^{1}$, Alexis Corral ${ }^{1}$, Axel Moreira ${ }^{2}$ and Alvaro Moreira $^{1 *}$ (D)
}

\begin{abstract}
Background: Inflammation plays an important role in the pathogenesis of many lung diseases. Preclinical studies suggest that mesenchymal stromal cell (MSC) conditioned media (CdM) can attenuate inflammation. Our aim was threefold: (1) summarize the existing animal literature evaluating $\mathrm{CdM}$ as a therapeutic agent for pediatric/adult lung disease, (2) quantify the effects of CdM on inflammation, and (3) compare inflammatory effects of CdM to MSCs.

Methods: Adhering to the Systematic Review Protocol for Animal Intervention Studies, a systematic search of English articles was performed in five databases. Meta-analysis and meta-regression were performed to generate random effect size using standardized mean difference (SMD).

Results: A total of 10 studies met inclusion. Lung diseases included bronchopulmonary dysplasia, asthma, pulmonary hypertension, and acute respiratory distress syndrome. CdM decreased inflammatory cells (1.02 SMD, $95 \% \mathrm{Cl} 0.86,1.18)$ and cytokines $(0.71 \mathrm{SMD}, 95 \% \mathrm{Cl} 0.59,0.84)$. The strongest effect for inflammatory cells was in bronchopulmonary dysplasia $(3.74 \mathrm{SMD}, 95 \% \mathrm{Cl} 3.13$, 4.36) while pulmonary hypertension had the greatest reduction in inflammatory cytokine expression (1.44 SMD, 95\% Cl 1.18, 1.71). Overall, CdM and MSCs had similar anti-inflammatory effects.

Conclusions: In this meta-analysis of animal models recapitulating lung disease, CdM improved inflammation and had an effect size comparable to MSCs. While these findings are encouraging, the risk of bias and heterogeneity limited the strength of our findings.
\end{abstract}

\section{Background}

Lung disease is a major cause of morbidity and mortality $[1,2]$. Respiratory conditions constitute 5 of the thirty most common causes of death worldwide [3]. As a result, the combined economic cost of respiratory disease in the UK in 2014 totaled $£ 11.1$ billion [4]. Thus, promoting healthy lives and minimizing lung infection/injury may preserve lifelong function and reduce chronic disease.

Inflammation plays a central role in the pathogenesis of many lung diseases [5-7]. An increasing number of studies have demonstrated a clear association between the degree of inflammation and lung structure/function

\footnotetext{
* Correspondence: MoreiraA@uthscsa.edu

${ }^{1}$ Department of Pediatrics, Division of Neonatology, University of Texas

Health-San Antonio, San Antonio, Texas 78229-3900, USA

Full list of author information is available at the end of the article
}

[8-10]. Even an acute phase of inflammation has been shown to alter airway gas exchange, its ability to repair injured epithelium, and clear excess alveolar fluid [11, 12]. Consequently, utilizing therapies that target airway inflammation is a promising approach to mitigate airway disease.

The pleotropic effects of mesenchymal stem/stromal cells (MSCs) have positioned them as novel agents against lung disease [13-16]. Multiple studies highlight the anti-inflammatory and restorative tissue effects of MSCs [17-19]. Previously, it was believed that the advantages of stem cells lay in their engrafting ability; however, research now demonstrates their beneficial effects are via secretion of paracrine factors [20-22]. These bioactive factors can be collectively obtained and harbor the ability of molecular cueing to suppress inflammation, improve wound healing, angiogenesis, and stimulate

(C) The Author(s). 2019 Open Access This article is distributed under the terms of the Creative Commons Attribution 4.0 International License (http://creativecommons.org/licenses/by/4.0/), which permits unrestricted use, distribution, and 
regeneration [23]. To date, it is unknown which MSC factor(s) mediate their biologic action but collecting the milieu of secreted factors in their conditioned media $(\mathrm{CdM})$ has proven successful in animal models of lung disease [24]. Advantages to the use of CdM over MSCs include biologic stability, cell-free transplantation, low risk of infection/recipient rejection, and ease for clinical scalability [25].

Currently, there has been no systematic review examining the therapeutic potential of MSC-derived CdM on inflammation in animal models recapitulating pediatric/ adult lung disease. The results of this study are intended to identify the current research gaps and to generalize methods for CdM research. This systematic review and meta-analysis aims to: (i) methodically review the current literature describing the effects of $\mathrm{CdM}$ on animal models of pediatric/adult lung disease, (ii) quantify and analyze the effect of $\mathrm{CdM}$ on inflammation, (iii) compare inflammatory effects of CdM to MSCs, and (iv) identify limitations/research gaps that should be addressed in future preclinical studies.

\section{Methods}

Our methods adhere to the guidelines established by the Systematic Review Centre for Laboratory Animal Experimentation (SYRCLE) and are described in Additional file 5: Table S1 [26]. Our protocol was registered through the Collaborative Approach to Meta-Analysis and Review of Data from Experimental Studies (CAMARADES) on August 7th, 2018.

\section{Literature search}

A literature search was performed using MEDLINE's database PubMed, Scopus, the Cumulative Index to Nursing and Allied Health Literature (CINAHL), Science Direct, and Google Scholar. Our major search criteria included "mesenchymal stem cell conditioned media," "lung disease," "animal," and their synonyms (refer to Additional file 6: Table S2 for full criteria). Screenings by title/abstract and then by full-text review were conducted independently by three investigators (AM, CE, and ED). Reference lists from the included studies and relevant reviews were used in an effort to obtain additional sources for inclusion.

\section{Inclusion and exclusion criteria}

In the current study, articles were included based on three main selection criteria: disease of interest, administration of MSC conditioned media, and assessment of inflammation. Specifically, in addressing disease, animal studies were included that focused on pediatric/adult models of bronchopulmonary dysplasia (BPD), asthma, pulmonary hypertension $(\mathrm{PH})$, acute respiratory distress syndrome (ARDS), cystic fibrosis (CF), and pneumonia.
Studies that addressed other pediatric or adult diseases were excluded. Of these, papers that addressed in vivo MSC-CdM administration were included, while papers solely addressing MSCs without CdM, or those using only ex vivo methods were excluded. Finally, articles were included only if their outcome assessment involved measures of inflammation. Papers with other outcome measures in addition to inflammation were still included.

\section{Primary and secondary endpoints}

We defined our primary endpoint as inflammation, which is reported through inflammatory marker analysis after administration of conditioned media treatment. The markers were measured from any animal lung tissue and were grouped as either immune cells or inflammatory cytokines/proteins. Studies were excluded from the selection process if data pertaining to our primary outcome could not be obtained or if the study was not done on in-vivo or in animal models.

Our secondary outcome of lung architecture and/or function were not required for inclusion but was reported in many of the selected studies. The type of architectural and functional measures varies between articles but are used as additional measurements to gauge the efficacy of the conditioned media treatment.

This review presents the results of the primary endpoint analysis, while results from the secondary endpoint analysis will be reported in a future paper.

\section{Data extraction}

Data was independently collected by two groups of investigators (ED and $\mathrm{CE} ; \mathrm{AC}$ and JM) and compared. A third investigator (AM) resolved any disagreements in the collected information. Extracted data included general study design (title, author, contact email, country, funding source(s), conflict(s) of interest), animal model characteristics (disease, disease induction, species, strain, age, gender, immune status), conditioned media characteristics (tissue source, dose, route of delivery, timing, frequency, CdM group name, disease group name), primary outcome measures (inflammatory markers and source, timing of outcome measurement), secondary outcome measures (lung architecture and/or lung function), other measures assessed, as well as a SYRCLE risk of bias assessment and MSC criteria assessment. Original data was gathered from graphs and plots using GetData graph digitizer version 2.26 when exact values were not accessible from the articles.

\section{Risk of bias}

Risk of bias was assessed independently by two investigators (RN and AM) per the 2014 SYRCLE Risk of Bias tool for animal studies. The tool provides ten categories 
that evaluate bias related to selection, performance, detection, attrition and reporting. The responses of "yes" demonstrates a low risk of bias and "no" indicates a high risk. Studies that did not explicitly state its methods were marked as "unclear."

\section{Data analysis}

Meta-analysis was conducted using a random effects model to generate forest plots. The estimated effect size of CdM or MSC on immune cells or inflammatory cytokine was determined using standardized mean difference (SMD) and a 95\% confidence interval (CI). Stratified effect was measured according to disease and by overall assessment of included lung disease.

Statistical heterogeneity between studies was calculated using the $\mathrm{I}^{2}$ metric. Potential sources of heterogeneity, if significant, were further investigated by meta-regression and subgroup analysis. Publication bias was examined using funnel plots with Egger's tests.

All statistical analyses were performed using STATA v.13 (College Station, TX, USA).

\section{Results}

\section{Study selection}

Our literature search produced 54 results from the utilized search terms. A total 49 results remained after removal of duplicate articles. After preliminary screening, 34 articles were excluded leaving 20 articles for fulltext review. From the remaining, 10 published articles met defined criteria set and reported our primary outcome of inflammatory markers (Fig. 1 and Additional file 7: Table S3). All studies were reported in the review and meta-analysis.

\section{Study characteristics}

The studies included in this review were published between the years 2009 and 2017. Six of the included studies were from the United States, two were from Iran, one from Canada, and another from Japan. Relevant characteristics of these articles are described in Table 1. All of the studies used rodents as their animal model, with $60 \%(n=6)$ incorporating either C57/BL6 or FVB mice. Gender was reported in $90 \%(n=9)$ of the studies, with the majority using only male rodents. Most of the studies used animals less than 12 weeks of age.

The pediatric/adult lung diseases that were represented in animal models included: asthma $(n=3)$, acute respiratory distress syndrome $(n=2)$, bronchopulmonary dysplasia $(\mathrm{n}=2)$, and pulmonary hypertension $(n=2)$. Most studies used a single dose of 30 or $50 \mu \mathrm{l}$ conditioned media, with an intravenous route being the most common delivery mode.

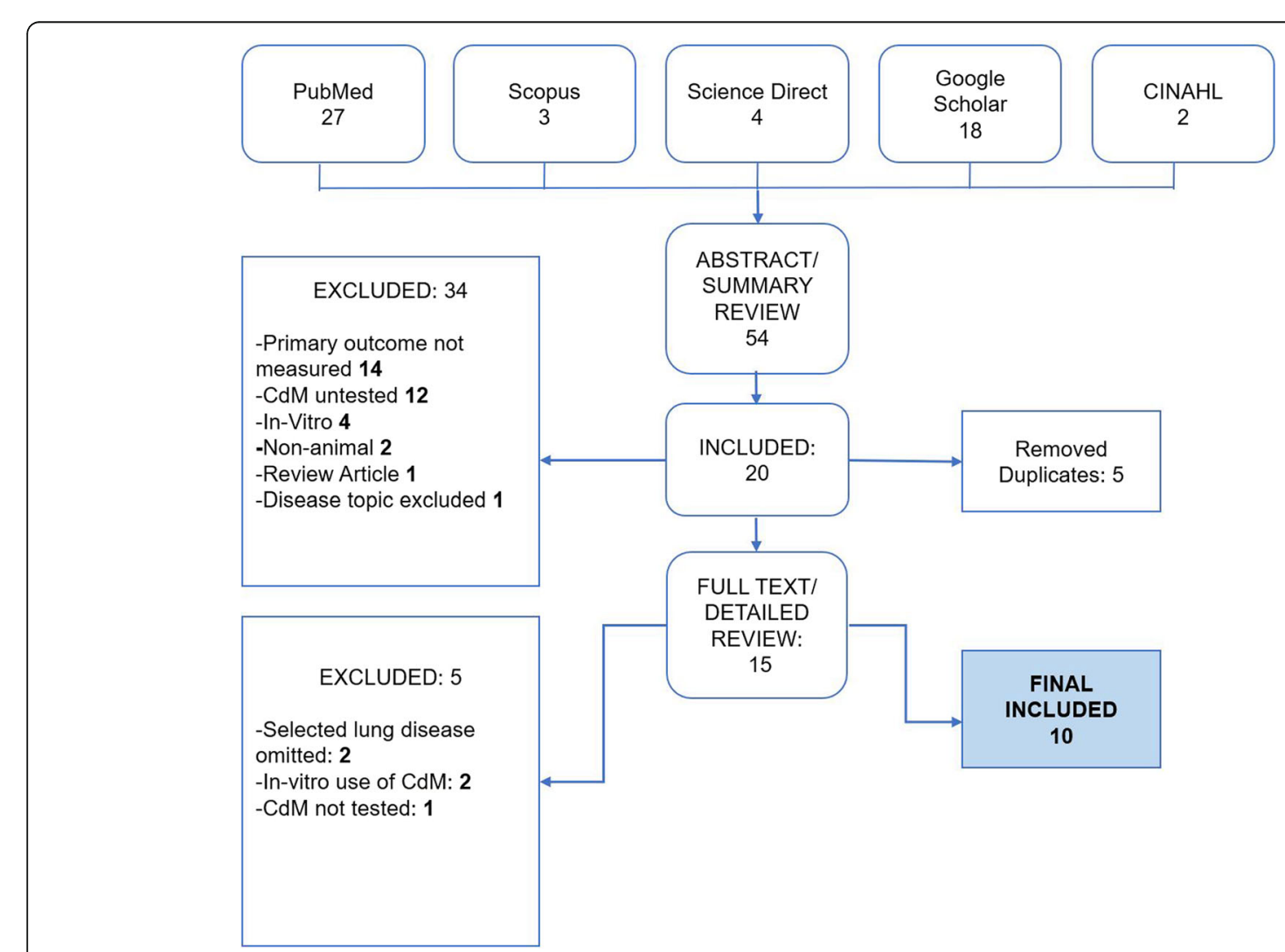

Fig. 1 Flow diagram demonstrating study selection process 
Table 1 Summary of study characteristics. Detailed summary of information extracted from included studies

\begin{tabular}{|c|c|c|c|c|c|c|c|c|}
\hline \multirow{3}{*}{$\begin{array}{l}\text { Author } \\
\text { (year) }\end{array}$} & \multicolumn{2}{|c|}{ Study Design } & \multicolumn{2}{|c|}{ Animal Characteristics } & \multicolumn{2}{|c|}{ Intervention Characteristics } & \multirow{3}{*}{$\begin{array}{l}\text { Primary } \\
\text { Outcome } \\
\text { Inflammation }\end{array}$} & \multirow{3}{*}{$\begin{array}{l}\text { Secondary } \\
\text { Outcomes } \\
\text { Lung } \\
\text { Architecture/ } \\
\text { Function }\end{array}$} \\
\hline & Disease & Disease & Animal & Age & Source; & Dose; & & \\
\hline & Model & Induction & Model; Gender & & (Origin) & $\begin{array}{l}\text { Delivery; Timing; } \\
\text { Frequency }\end{array}$ & & \\
\hline $\begin{array}{l}\text { lonescu } \\
\text { (2012) }\end{array}$ & ARDS & $\begin{array}{l}\text { IT injection of } 4 \\
\mathrm{mg} / \mathrm{kg} \text { LPS } \\
\text { (E. coli 055:B5) }\end{array}$ & $\begin{array}{l}\text { C57/BL6 } \\
\text { mice; Male }\end{array}$ & Adult & $\begin{array}{l}\text { Bone } \\
\text { Marrow }\end{array}$ & $\begin{array}{l}30 \mu \mathrm{l} ; \mathrm{IT} ; 4 \mathrm{~h} \\
\text { post-LPS } \\
\text { exposure; once }\end{array}$ & Cytokines & Lung sections \\
\hline Lu (2015) & ARDS & $\begin{array}{l}\text { Oropharyngeal } \\
\text { aspiration } 2 \text { mg/kg } \\
\text { LPS (E. Coli 0127:B8) }\end{array}$ & $\begin{array}{l}\text { C57/BL6 } \\
\text { mice; Male }\end{array}$ & $\begin{array}{l}\text { Not } \\
\text { Reported }\end{array}$ & $\begin{array}{l}\text { Adipose } \\
\text { Tissue }\end{array}$ & $\begin{array}{l}200 \mu \mathrm{\mu l} \text {; IV tail } \\
\text { vein; } 4 \mathrm{~h} \text { post-LPS } \\
\text { exposure; once }\end{array}$ & $\begin{array}{l}\text { TNF-alpha; } \\
\text { IL-6; MIP-2; } \\
\text { IL-10; VEGF }\end{array}$ & $\begin{array}{l}\text { Alveolar wall } \\
\text { sections }\end{array}$ \\
\hline $\begin{array}{l}\text { Wakaya- } \\
\text { ma (2015) }\end{array}$ & ARDS & $6 \mathrm{U} / \mathrm{kg}$ Bleomycin & $\begin{array}{l}\text { C57/BL6J } \\
\text { mice; Female }\end{array}$ & Adult & $\begin{array}{l}\text { Human } \\
\text { Exfoliated } \\
\text { Deciduous } \\
\text { Teeth }\end{array}$ & $\begin{array}{l}500 \mu \mathrm{ll} \text {; IV jugular } \\
\text { vein; } 24 \mathrm{~h} \text { post- } \\
\text { bleomycin exposure; } \\
\text { once }\end{array}$ & $\begin{array}{l}\text { Cell surface } \\
\text { markers; } \\
\text { Cytokines }\end{array}$ & $\begin{array}{l}\text { Fibrotic lung } \\
\text { sections }\end{array}$ \\
\hline $\begin{array}{l}\text { Ahmadi } \\
\text { (2016) }\end{array}$ & Asthma & $\begin{array}{l}\text { IP administered OVA } \\
\text { on days } 1 \text { and } 8 \text {; day } \\
14 \text {, exposure to } 4 \% \\
\text { OVA nebulizer for } 5 \\
\text { min for } 18+/-1 \text { days }\end{array}$ & $\begin{array}{l}\text { Wistar rats; } \\
\text { Male }\end{array}$ & Adult & $\begin{array}{l}\text { Bone } \\
\text { Marrow }\end{array}$ & $\begin{array}{l}50 \mu \text { l; IV left } \\
\text { femoral vein; } \\
1 \text { day post } \\
\text { sensitization; } \\
\text { once }\end{array}$ & $\begin{array}{l}\text { CD3+; CD4+; } \\
\text { CD8+ }\end{array}$ & \\
\hline $\begin{array}{l}\text { Ahmadi } \\
\text { (2017) }\end{array}$ & Asthma & $\begin{array}{l}\text { IP administered OVA } \\
\text { on days } 1 \text { and } 8 \text {; day } \\
14,5 \text { min exposure } \\
\text { to } 4 \% \text { OVA nebulizer } \\
\text { for } 18+/-1 \text { days }\end{array}$ & $\begin{array}{l}\text { Wistar rats; } \\
\text { Male }\end{array}$ & Adult & $\begin{array}{l}\text { Bone } \\
\text { Marrow }\end{array}$ & $\begin{array}{l}50 \mu \mathrm{l} ; \mathrm{IT} ; 1 \text { day } \\
\text { post sensitization; } \\
\text { once }\end{array}$ & IL-4; IL-10 & $\begin{array}{l}\text { Emphysema; } \\
\text { Atelectasis; } \\
\text { Hyperemia; } \\
\text { Epithelialization; } \\
\text { Leukocyte } \\
\text { Infiltration }\end{array}$ \\
\hline $\begin{array}{l}\text { Cruz } \\
(2015)\end{array}$ & Asthma & $\begin{array}{l}5 \mu \mathrm{g} \text { Aspergillus } \\
\text { fumigatus days } 0 \text { and } \\
7 \text { for sensitization; } \\
\text { same inoculation on } \\
\text { days } 14 \text { and } 16 \text { for } \\
\text { challenge }\end{array}$ & $\begin{array}{l}\text { C57/BL6 mice; } \\
\text { Male }\end{array}$ & Adult & $\begin{array}{l}\text { Bone } \\
\text { Marrow }\end{array}$ & $\begin{array}{l}200 \mu \mathrm{\mu l} \text {; IV tail vein; } \\
\text { day } 14 \text { after aspergillus } \\
\text { challenge; once }\end{array}$ & $\begin{array}{l}\text { Neutrophils; } \\
\text { Eosinophils; } \\
\text { Macrophages; } \\
\text { Lymphocytes; } \\
\text { Cytokines }\end{array}$ & $\begin{array}{l}\text { Lung sections; } \\
\text { Airway resistance; } \\
\text { Tissue resistance; } \\
\text { lung elasticity }\end{array}$ \\
\hline $\begin{array}{l}\text { Aslam } \\
(2009)\end{array}$ & BPD & $\begin{array}{l}\text { Exposure to } 75 \% \\
\mathrm{O}_{2} \text { within } 24 \mathrm{~h} \\
\text { of birth }\end{array}$ & $\begin{array}{l}\text { FVB mice; } \\
\text { male/female }\end{array}$ & Neonate & $\begin{array}{l}\text { Bone } \\
\text { Marrow }\end{array}$ & $\begin{array}{l}50 \mu \text { l; IV superficial } \\
\text { temporal vein; } \\
\text { postnatal day 4; } \\
\text { once }\end{array}$ & $\begin{array}{l}\text { Macrophage; } \\
\text { Neutrophils, } \\
\text { Cytokines }\end{array}$ & $\begin{array}{l}\text { Pulmonary } \\
\text { arteriole; } \\
\text { Lung alveoli; } \\
\text { Medial } \\
\text { wall thickness }\end{array}$ \\
\hline $\begin{array}{l}\text { Sutsko } \\
(2012)\end{array}$ & BPD & $\begin{array}{l}2 \text { weeks } 90 \% \mathrm{O}_{2} \\
\text { exposure }\end{array}$ & $\begin{array}{l}\text { Sprague- } \\
\text { Dawley rats; } \\
\text { male/female }\end{array}$ & Neonate & $\begin{array}{l}\text { Bone } \\
\text { Marrow }\end{array}$ & $\begin{array}{l}50 \mu \mathrm{\mu l} \text {; IT; postnatal } \\
\text { day 9; once }\end{array}$ & $\begin{array}{l}\text { IL-6/18 s; IL-1B/ } \\
18 \mathrm{~s} ; \text { TTF-1/18 s; } \\
\text { VEGF; } \\
\text { angiopoietin-1 }\end{array}$ & $\begin{array}{l}\text { Alveolarization; } \\
\text { lung vascular } \\
\text { density }\end{array}$ \\
\hline $\begin{array}{l}\text { Lee } \\
\text { (2012) }\end{array}$ & $\mathrm{PH}$ & $\begin{array}{l}8.5 \pm 0.5 \% \mathrm{O}_{2} \\
\text { exposure for } 48 \mathrm{~h}\end{array}$ & $\begin{array}{l}\text { FVB mice; } \\
\text { gender not } \\
\text { reported }\end{array}$ & Adult & $\begin{array}{l}\text { Bone } \\
\text { Marrow }\end{array}$ & $\begin{array}{l}50 \mu \text { l; IV left jugular } \\
\text { vein; before } 48 \mathrm{~h} \\
\text { hypoxia induction; } \\
\text { once }\end{array}$ & $\begin{array}{l}\text { MCP-1; FIZZ-1/ } \\
\text { HIMF }\end{array}$ & \\
\hline $\begin{array}{l}\text { Rathinas- } \\
\text { abapathy } \\
\text { (2016) }\end{array}$ & $\mathrm{PH}$ & $\begin{array}{l}50 \mathrm{mg} / \mathrm{kg} \\
\text { Monocrotaline }\end{array}$ & $\begin{array}{l}\text { Sprague-Dawley } \\
\text { rats; Male }\end{array}$ & Adult & $\begin{array}{l}\text { Adipose } \\
\text { Tissue }\end{array}$ & $\begin{array}{l}100 \mu \text { l; IV jugular vein; } \\
14 \text { days post-MCT } \\
\text { exposure; once }\end{array}$ & $\begin{array}{l}\text { Cell surface } \\
\text { markers; } \\
\text { Cytokines }\end{array}$ & $\begin{array}{l}\text { Pulmonary vessel } \\
\text { wall thickness; } \\
\text { RV fibrosis }\end{array}$ \\
\hline
\end{tabular}

Inflammation was most commonly assessed via cytokine assays. A number of studies also investigated inflammation by flow cytometry. Eight of the articles examined secondary outcomes of interest.

\section{Conditioned media characteristics}

Conditioned media criteria of the included studies are summarized in Additional file 8: Table S4. Allogeneic bone marrow tissue was the most common source of MSCs (70\%). Six of the studies used Dulbecco's Modified
Eagle Medium for cell expansion and eight included antibiotic solution in their media.

\section{Risk of bias}

Risk of bias was assessed via SYRCLE Risk of Bias tool for all ten studies included in our review. No study was judged as low risk across all ten domains. Only two stated that the allocation selection was random, thus many were evaluated as being "unclear" for sequence generation bias. The majority of experimental and 
control groups were reported to be similar at baseline, though two were deemed high risk for inconsistencies between groups. Ten percent $(n=1)$ of studies concealed allocation of treatment, whereas the rest did not explicitly state allocation method and were deemed "unclear". None of the studies randomly housed the animals. Almost half $(n=4)$ of the studies randomly selected animals for outcome assessment, but only two studies reported blinding the outcome assessor. All studies were found to sufficiently report complete data and only one study was found to have reporting bias. Refer to Table 2 .

\section{Stratified meta-analysis: Inflammatory outcomes Immune Cells \& CdM}

Of the ten articles, seven evaluated the effect of CdM on immune cells. These studies incorporated the following pediatric/adult lung diseases: asthma, BPD, ARDS, and $\mathrm{PH}$. An overall improvement in inflammatory cell recruitment occurred after CdM therapy with an SMD of 1.02 (95\% CI 0.86, 1.18; 7 studies and 61 comparisons; Fig. 2). However, the heterogeneity between groups was significant $\left(\mathrm{I}^{2}=80.9 \% ; p<0.001\right)$. When stratified and assessed by disease, conditioned media was favored over control for each disease but performed best for bronchopulmonary dysplasia (3.74 SMD; 95\% CI 3.13, 4.36) followed by pulmonary hypertension (2.42 SMD; 95\% CI 1.32,3.52). Administration of $\mathrm{CdM}$ in asthma animal models decreased eosinophilia but did not have much effect on lymphocytes. The total number of cells in the bronchoalveolar lavage of asthma and ARDS animals was reduced with CdM.

Stratification by animal model, study design, and intervention characteristics revealed significant differences in effect size for immune cells, seen in Table 3. Effect size was largest in BPD, studies conducted in mice (1.61 SMD; 95\% CI 1.40, 1.82) and animals at neonatal age
(3.74 SMD; 95\% CI 3.13, 4.36). For intervention characteristics, effect size was larger in bone marrow derived conditioned media (1.10 SMD; 95\% CI 0.93, 1.27). Also, effect size was larger when intervention was delivered intravenously (1.19 SMD; 95\% CI 1.00, 1.39) and given greater than 72 -h after injury (2.30 SMD; $95 \%$ CI 2.01, 2.59).

\section{Cytokines \& CdM}

Of the ten articles, seven evaluated the effect of CdM on inflammatory cytokines. The studies included animal models mimicking asthma, BPD, ARDS, and PH. Like the outcomes in immune cells, CdM had an overall positive effect on cytokine analysis (SMD of $0.7195 \% \mathrm{CI}$, 0.59, 0.84; Fig. 3). Significant heterogeneity $\left(\mathrm{I}^{2}=81.9 \%\right.$; $p<0.001)$ was observed. When assessed by disease, conditioned media decreased cytokine expression for each disease except for bronchopulmonary dysplasia. Among the cytokines, CdM lessened the expression of tumor necrosis factor alpha, interleukin beta, transforming growth factor beta, and increased anti-inflammatory factors interleukin 10. In asthma preclinical studies, the treatment effect shifted away from a $\mathrm{T}$ cell helper 2 biased inflammation (e.g. interleukin 5, 4, and interferon gamma) [27]. Stratification by animal model, study design, and intervention characteristics were also performed for the assessment of cytokines, seen in Table 4 . Effect size was largest for pulmonary hypertension (1.44 SMD; 95\% CI 1.18, 1.71). The effect size was highest in mice $(1.22$ SMD; 95\% CI 0.97, 1.47) and male animals (1.02 SMD; 95\% CI 0.86, 1.18). For intervention characteristics, effect size was larger in LPS induced injury models (1.56 SMD; 95\% CI 0.82, 2.31). Adipose tissue derived conditioned media (1.46 SMD; 95\% CI 1.21, 1.71) had larger effect sizes at a dose of $100 \mu \mathrm{L}$. Similar to the immune cell assessment, intravenously delivered

Table 2 SYRCLE Risk of Bias Assessment for included studies

\begin{tabular}{|c|c|c|c|c|c|c|c|c|c|c|}
\hline Author (Year) & $\begin{array}{l}\text { Random } \\
\text { sequence } \\
\text { generation? }\end{array}$ & $\begin{array}{l}\text { Groups } \\
\text { similar at } \\
\text { baseline? }\end{array}$ & $\begin{array}{l}\text { Allocation } \\
\text { concealed? }\end{array}$ & $\begin{array}{l}\text { Animals } \\
\text { randomly } \\
\text { housed? }\end{array}$ & $\begin{array}{l}\text { Blinding of } \\
\text { caregivers } \\
\text { and/or } \\
\text { examiners? }\end{array}$ & $\begin{array}{l}\text { Random } \\
\text { selection for } \\
\text { outcome } \\
\text { assessment? }\end{array}$ & $\begin{array}{l}\text { Blinding } \\
\text { of } \\
\text { outcome } \\
\text { assessor? }\end{array}$ & $\begin{array}{l}\text { Incomplete } \\
\text { outcome } \\
\text { data } \\
\text { addressed? }\end{array}$ & $\begin{array}{l}\text { Free from } \\
\text { selective } \\
\text { outcome } \\
\text { reporting? }\end{array}$ & $\begin{array}{l}\text { Free } \\
\text { from } \\
\text { other } \\
\text { bias? }\end{array}$ \\
\hline Ahmadi (2017) & Yes & Yes & Unclear & Unclear & Unclear & Yes & Unclear & Yes & Yes & Yes \\
\hline Ahmadi (2016) & Yes & Yes & Unclear & Unclear & Unclear & Unclear & Unclear & Yes & Yes & Yes \\
\hline Aslam (2009) & Unclear & No & Unclear & Unclear & Unclear & Unclear & Unclear & Yes & Yes & Yes \\
\hline Cruz (2015) & Unclear & Yes & Unclear & Unclear & Unclear & Unclear & Unclear & Yes & No & Yes \\
\hline Ionescu (2012) & Unclear & Yes & Unclear & Unclear & Unclear & Unclear & Unclear & Yes & Yes & Yes \\
\hline Lee (2012) & Unclear & Unclear & Unclear & Unclear & Unclear & Unclear & Unclear & Yes & Yes & Yes \\
\hline Lu (2015) & Unclear & Yes & Unclear & Unclear & Unclear & Unclear & Unclear & Yes & Yes & Yes \\
\hline $\begin{array}{l}\text { Rathinasabapathy } \\
\text { (2016) }\end{array}$ & Unclear & Yes & Yes & Unclear & Yes & Yes & Yes & Yes & Yes & Yes \\
\hline Sutsko (2012) & Unclear & No & Unclear & Unclear & Unclear & Yes & Yes & Yes & Yes & Yes \\
\hline Wakayama (2015) & Unclear & Yes & Unclear & Unclear & Unclear & Yes & Unclear & Yes & Yes & Yes \\
\hline
\end{tabular}




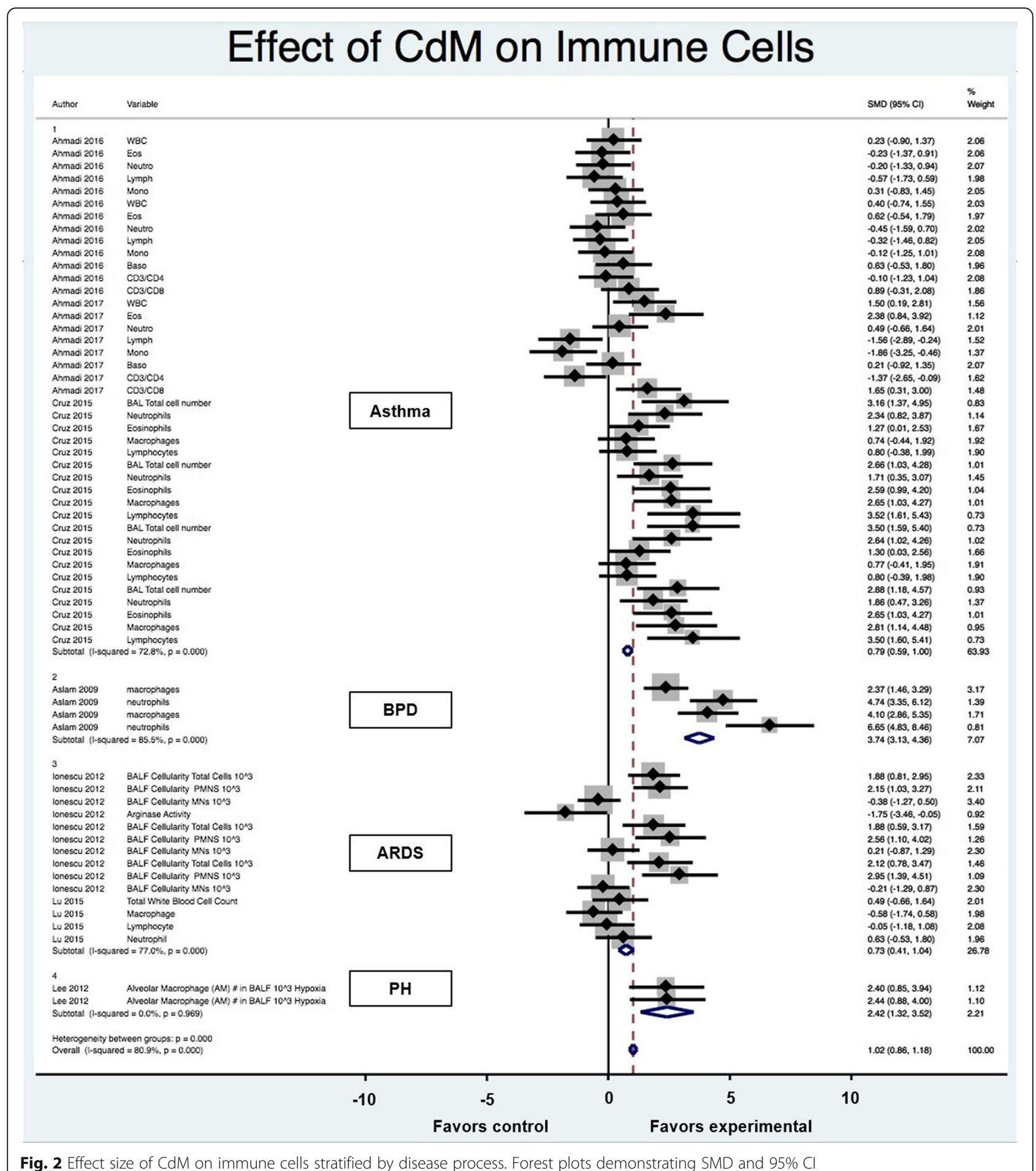

Fig. 2 Effect size of CdM on immune cells stratified by disease process. Forest plots demonstrating SMD and 95\% Cl

intervention (0.73 SMD; 95\% CI 0.60, 0.85), and delivery greater than 72-h after injury (0.92 SMD; 95\% CI 0.77, 1.07) had the largest effect size.

\section{Immune Cells \& MSCs}

From the ten articles, six evaluated the effects of MSC on immune cells (Fig. 4). The studies highlighted lung disease in animal models of asthma, bronchopulmonary dysplasia, and acute respiratory distress syndrome. MSCs had an overall positive effect and was favored over the control with a SMD of 1.26 (95\% CI 1.08, 1.43). However, the heterogeneity between groups was significant $\left(\mathrm{I}^{2}=\right.$ 81.5\%; $p<0.001)$. When assessed for each disease, 
Table 3 Stratification of estimated CdM effect size on immune cells

\begin{tabular}{|c|c|c|c|c|c|c|c|}
\hline & \# Studies & \# Comparisons & SMD (95\% Cl) & $\%$ Weight & $1 \wedge 2$ & $p^{*}$ & $p^{* *}$ \\
\hline \multicolumn{8}{|l|}{ Disease } \\
\hline Asthma & 3 & 41 & $0.79(0.59,0.10)$ & 63.93 & 72.80 & 0.00 & \multirow[t]{4}{*}{0.59} \\
\hline BPD & 1 & 4 & $3.74(3.13,4.36)$ & 7.07 & 85.50 & 0.00 & \\
\hline ARDS & 2 & 14 & $0.72(0.41,1.04)$ & 26.78 & 77.00 & 0.00 & \\
\hline $\mathrm{PH}$ & 1 & 2 & $2.42(1.32,3.52)$ & 2.21 & 0.00 & 0.97 & \\
\hline \multicolumn{8}{|l|}{ Animal model/species } \\
\hline Mouse & 5 & 40 & $1.61(1.40,1.82)$ & 60.98 & 79.60 & 0.00 & \multirow[t]{2}{*}{0.00} \\
\hline Rat & 2 & 21 & $0.10(-0.17,0.36)$ & 39.02 & 54.90 & 0.00 & \\
\hline \multicolumn{8}{|l|}{ Strain } \\
\hline C57/BL6 & 4 & 36 & $1.33(1.11,1.56)$ & 53.91 & 70.30 & 0.00 & \multirow[t]{3}{*}{0.00} \\
\hline FVB & 1 & 4 & $3.74(3.13,4.36)$ & 7.07 & 85.50 & 0.00 & \\
\hline Wistar & 2 & 21 & $0.10(-0.17,0.36)$ & 39.02 & 54.90 & 0.00 & \\
\hline \multicolumn{8}{|l|}{ Gender } \\
\hline Male & 5 & 55 & $0.77(0.60,0.95)$ & 90.71 & 73.50 & 0.00 & \multirow[t]{3}{*}{0.01} \\
\hline Mixed & 1 & 4 & $3.74(3.13,4.36)$ & 7.07 & 85.50 & 0.00 & \\
\hline Not reported & 1 & 2 & $2.42(1.32,3.52)$ & 2.21 & 0.00 & 0.97 & \\
\hline \multicolumn{8}{|l|}{ Age } \\
\hline Neonatal & 1 & 4 & $3.74(3.13,4.36)$ & 7.07 & 85.50 & 0.00 & \multirow[t]{3}{*}{0.00} \\
\hline Adult & 5 & 53 & $0.80(0.70,1.06)$ & 84.90 & 74.50 & 0.00 & \\
\hline Not reported & 1 & 4 & $0.12(-0.46,0.70)$ & 8.03 & 0.00 & 0.45 & \\
\hline \multicolumn{8}{|c|}{ Intervention characteristics } \\
\hline Asthma & & & & & & & 0.18 \\
\hline Ovalbumin & 2 & 21 & $0.10(-0.17,0.36)$ & 39.02 & 54.90 & 0.00 & \\
\hline Aspergillus exposure & 1 & 19 & $1.89(1.56,2.21)$ & 24.91 & 41.60 & 0.03 & \\
\hline \multicolumn{8}{|l|}{ ARDS } \\
\hline LPS & 2 & 14 & $0.73(.41,1.04)$ & 26.78 & 77.00 & 0.00 & \\
\hline \multicolumn{8}{|l|}{$B P D$} \\
\hline 75\% hyperoxia & 1 & 4 & $3.74(3.13,4.36)$ & 7.07 & 85.50 & 0.00 & \\
\hline \multicolumn{8}{|l|}{$P H$} \\
\hline Hypoxia & 1 & 2 & $2.42(1.32,3.52)$ & 2.21 & 0.00 & 0.97 & \\
\hline \multicolumn{8}{|l|}{ Source } \\
\hline Bone marrow & 6 & 57 & $1.10(0.93,1.27)$ & 91.97 & 81.40 & 0.00 & \\
\hline Adipose tissue & 1 & 4 & $0.12(-0.46,0.70)$ & 8.03 & 0.00 & 0.45 & 0.14 \\
\hline \multicolumn{8}{|l|}{ Origin } \\
\hline Allogenic & 7 & 61 & $1.02(0.86,1.18)$ & 100.00 & 80.90 & 0.00 & 0.00 \\
\hline \multicolumn{8}{|l|}{ Dose } \\
\hline 200ul & 2 & 24 & $1.46(1.17,1.74)$ & 32.95 & 63.20 & 0.00 & \multirow[t]{3}{*}{0.00} \\
\hline 50ul & 4 & 27 & $0.74(0.50,0.97)$ & 48.30 & 86.30 & 0.00 & \\
\hline $30 u l$ & 1 & 10 & $0.99(1.17,1.74)$ & 18.75 & 81.20 & 0.00 & \\
\hline \multicolumn{8}{|l|}{ Delivery } \\
\hline Intravenous & 5 & 43 & $1.19(1.00,1.39)$ & 68.50 & 80.20 & 0.00 & \multirow[t]{2}{*}{0.08} \\
\hline Intratracheal & 2 & 18 & $0.64(0.35,0.93)$ & 31.50 & 81.70 & 0.00 & \\
\hline \multicolumn{8}{|l|}{ Timing of treatment } \\
\hline$<72 \mathrm{hr}$ & 5 & 37 & $0.42(0.22,0.62)$ & 68.01 & 70.80 & 0.00 & \multirow[t]{2}{*}{0.00} \\
\hline$>72 \mathrm{hr}$ & 2 & 24 & $2.30(2.01,2.59)$ & 31.99 & 71.40 & 0.00 & \\
\hline
\end{tabular}




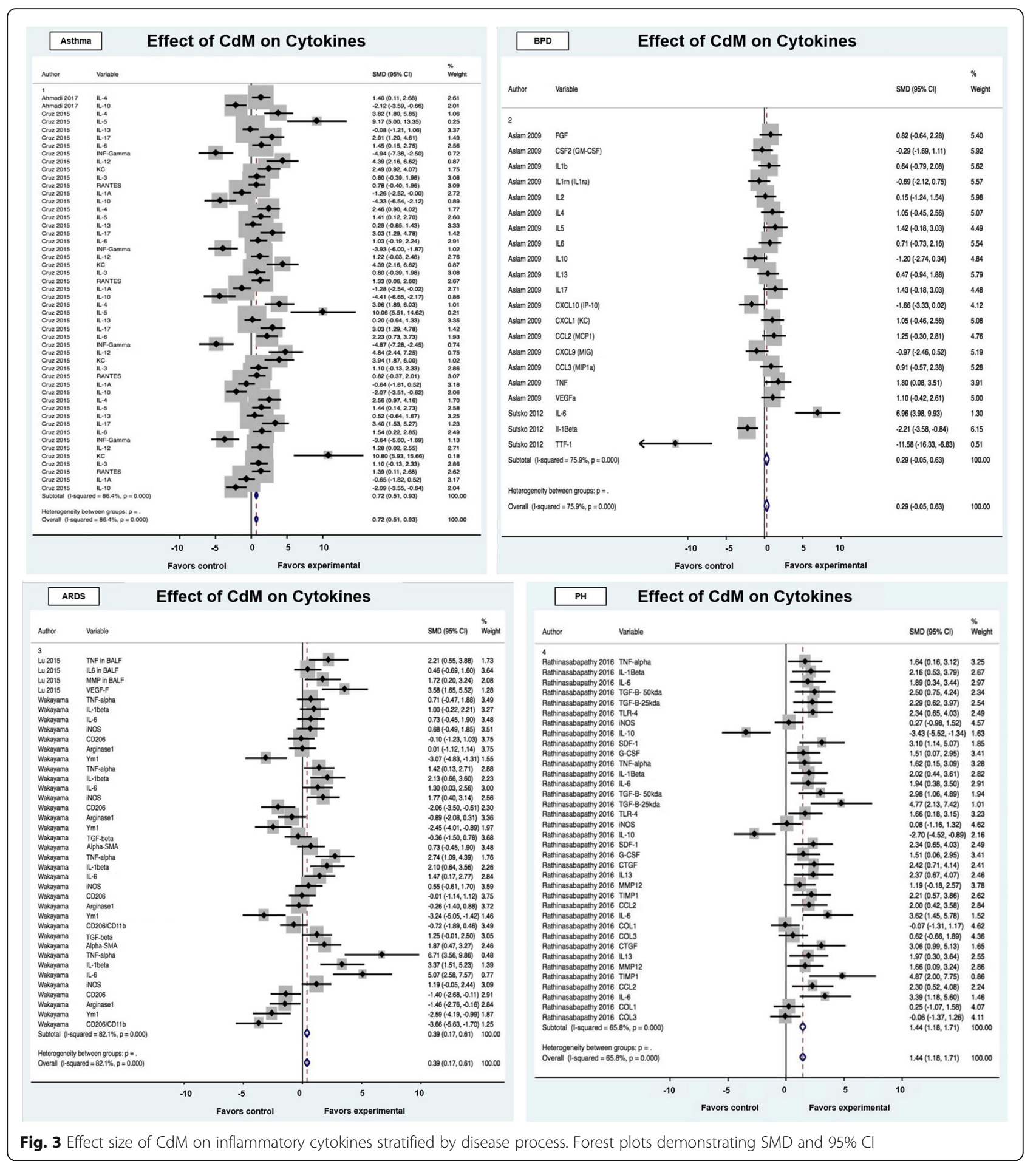

the MSC treatment was favored over control for each model but performed best for bronchopulmonary dysplasia (3.14 SMD; 95\% CI 2.60, 3.67). Administration of MSCs in asthma animal models decreased eosinophilia, neutrophilia and overall WBC. The total number of cells in the bronchoalveolar lavage was decreased in all studies.
Cytokines \& MSCS

Five of the ten articles assessed the effect of MSCs on inflammatory cytokines and included animal models of the following lung diseases: asthma, BPD, ARDS, and $\mathrm{PH}$ (Fig. 5). The effect of the MSCs on the injury model evaluated via cytokine analysis was positive overall with an SMD of $1.00(95 \%$ CI $0.85,1.15)$. Significant 
Table 4 Stratification of estimated CdM effect size on inflammatory cytokines

\begin{tabular}{|c|c|c|c|c|c|c|c|}
\hline \multicolumn{8}{|l|}{ Disease } \\
\hline Asthma & 2 & 50 & $0.72(0.51,0.93)$ & 34.70 & 86.40 & 0 & \multirow[t]{4}{*}{0.29} \\
\hline BPD & 2 & 21 & $0.29(-0.05,0.63)$ & 13.00 & 75.90 & 0 & \\
\hline ARDS & 2 & 38 & $0.39(0.17,0.61)$ & 31.19 & 82.10 & 0 & \\
\hline $\mathrm{PH}$ & 1 & 36 & $1.44(1.18,1.71)$ & 21.11 & 65.80 & 0 & \\
\hline \multicolumn{8}{|l|}{ Animal model/species } \\
\hline Mouse & 4 & 104 & $1.22(0.97,1.47)$ & 23.75 & 82.40 & 0 & \multirow[t]{2}{*}{0.13} \\
\hline Rat & 3 & 41 & $0.56(0.42,0.70)$ & 76.25 & 79.10 & 0 & \\
\hline \multicolumn{8}{|l|}{ Strain } \\
\hline C57/BL6 & 3 & 86 & $0.58(0.43,0.73)$ & 64.29 & 84.80 & 0 & \multirow[t]{4}{*}{0.29} \\
\hline FVB & 2 & 18 & $0.43(0.07,0.78)$ & 11.97 & 36.00 & 0.07 & \\
\hline Sprague-Dawley & 1 & 39 & $1.32(1.06,1.58)$ & 22.14 & 77.70 & 0 & \\
\hline Wistar & 1 & 2 & $(-) 0.14(-1.10,0.83)$ & 1.60 & 92.00 & 0 & \\
\hline \multicolumn{8}{|l|}{ Gender } \\
\hline Male & 4 & 90 & $1.02(0.86,1.18)$ & 58.53 & 81.90 & 0 & \multirow[t]{3}{*}{0.03} \\
\hline Mixed & 2 & 21 & $0.29(-0.05,0.63)$ & 13.00 & 75.90 & 0 & \\
\hline Female & 1 & 34 & $0.28(0.05,0.51)$ & 28.47 & 82.50 & 0 & \\
\hline \multicolumn{8}{|l|}{ Age } \\
\hline Neonatal & 1 & 21 & $0.29(-0.05,0.63)$ & 13.00 & 75.90 & 0 & \multirow[t]{3}{*}{0.13} \\
\hline Adult & 6 & 120 & $0.75(0.62,0.89)$ & 84.27 & 92.00 & 0 & \\
\hline NR & 1 & 4 & $1.56(0.82,2.31)$ & 2.73 & 64.20 & 0.04 & \\
\hline \multicolumn{8}{|l|}{ Intervention characteristics } \\
\hline Asthma & & & & & & & \multirow[t]{3}{*}{0.26} \\
\hline Ovalbumin & 1 & 2 & $(-) 0.14(-1.10,0.83)$ & 1.60 & 92.00 & 0 & \\
\hline Aspergillus exposure & 1 & 48 & $0.76(0.55,0.98)$ & 33.09 & 86.40 & 0 & \\
\hline \multicolumn{8}{|l|}{ ARDS } \\
\hline LPS & 1 & 4 & $1.56(0.82,2.31)$ & 2.73 & 64.20 & 0.04 & \\
\hline Bleomycin & 1 & 34 & $0.28(0.05,0.51)$ & 28.47 & 82.50 & 0 & \\
\hline \multicolumn{8}{|l|}{$B P D$} \\
\hline 75\% hyperoxia & 1 & 18 & $0.43(0.07,0.78)$ & 11.97 & 36.00 & 0.07 & \\
\hline $90 \%$ hyperoxia & 1 & 3 & $(-) 1.31(-2.51,-0.10)$ & 1.03 & 95.70 & 0 & \\
\hline \multicolumn{8}{|l|}{$\mathrm{PH}$} \\
\hline Moncrotaline/bleomycin & 1 & 36 & $1.44(1.18,1.71)$ & 21.11 & 65.80 & 0 & \\
\hline \multicolumn{8}{|l|}{ Source } \\
\hline Bone marrow & 4 & 71 & $0.60(0.43,0.78)$ & 47.70 & 84.40 & 0 & \multirow[t]{3}{*}{0.42} \\
\hline Adipose tissue & 2 & 40 & $1.46(1.21,1.71)$ & 23.83 & 64.80 & 0 & \\
\hline Decidious teeth & 1 & 34 & $0.28(0.05,0.51)$ & 28.47 & 82.50 & 0 & \\
\hline \multicolumn{8}{|l|}{ Origin } \\
\hline Allogenic & 6 & 111 & $0.89(0.74,1.03)$ & 71.53 & 81.30 & 0 & \multirow[t]{2}{*}{0.11} \\
\hline Xenogenic & 1 & 34 & $0.28(0.05,0.51)$ & 28.47 & 82.50 & 0 & \\
\hline \multicolumn{8}{|l|}{ Dose } \\
\hline $500 u l$ & 2 & 34 & $0.28(0.05,0.51)$ & 28.47 & 82.50 & 0 & \multirow[t]{4}{*}{0.63} \\
\hline $200 u l$ & 1 & 52 & $0.82(0.62,1.03)$ & 35.82 & 85.80 & 0 & \\
\hline 100ul & 1 & 36 & $1.44(1.18,1.71)$ & 21.11 & 65.80 & 0 & \\
\hline 50ul & 3 & 23 & $0.24(-0.08,0.56)$ & 14.61 & 77.20 & 0 & \\
\hline
\end{tabular}


Table 4 Stratification of estimated CdM effect size on inflammatory cytokines (Continued)

\begin{tabular}{|c|c|c|c|c|c|c|c|}
\hline Disease & & & & & & & \\
\hline \multicolumn{8}{|l|}{ Delivery } \\
\hline Intravenous & 6 & 143 & $0.73(0.60,0.85)$ & 98.40 & 81.80 & 0 & 0.45 \\
\hline Intratracheal & 1 & 2 & $(-) 0.14(-1.10,0.83)$ & 1.60 & 92.00 & 0 & \\
\hline \multicolumn{8}{|l|}{ Timing } \\
\hline$<72 \mathrm{~h}$ & 3 & 43 & $0.31(0.10,0.52)$ & 33.83 & 84.90 & 0 & 0.08 \\
\hline$>72 \mathrm{~h}$ & 4 & 102 & $0.92(0.77,1.07)$ & 66.17 & 79.80 & 0 & \\
\hline
\end{tabular}

heterogeneity $\left(\mathrm{I}^{2}=78.7 \% ; p<0.001\right)$ was observed. The intervention was most effective for pulmonary hypertension (1.44 SMD; 95\% CI 1.18, 1.71). Among the cytokines, MSCs reduced the expression of interleukin 6, interferon gamma, and increased interleukin 10. In bronchopulmonary dysplasia, MSC treatment improved the expression of thyroid transcription factor 1, a gene important in normal lung embryogenesis and surfactant production [28]. Like CdM effects in asthma, MSCs shifted away from a $\mathrm{T}$ cell helper 2 biased inflammation.

\section{CdM vs MSC comparison}

Immune cells: CdM v. MSCs

In assessing the overall effects on immune cells in asthma, BPD, and ARDS, the effectiveness of CdM vs MSC treatment was comparable [SMD of -0.15 (95\% CI $-0.31,0.01)]$. Graphical representation available in Additional file 1: Figure S1. Asthma slightly favored CdM but BPD and ARDS were slightly improved after MSC treatment.

\section{Cytokines: CdM v. MSCs}

In evaluating treatment effect on cytokines, overall assessment showed similar results between CdM vs MSC [SMD of $-0.10(95 \%$ CI $-0.24,0.04)]$. Again, asthma favored CdM but MSC therapy was better for BPD and PH (Additional file 2: Figure S2).

\section{Subgroup analysis in ARDS}

ARDS was the only disease that had differing tissue sources of CdM (bone marrow vs. adipose vs. decidual teeth) with more than one comparison study for immune cells and inflammatory cytokines. Additional file 3: Figure S3 shows that bone marrow CdM had a greater reduction in inflammatory cells when compared to adipose-derived CdM (SMD of 0.99 vs. SMD of 0.12) In contrast, adipose-generated CdM was superior to decidual teeth $\mathrm{CdM}$ in respects to inflammatory cytokines (SMD of 1.56 vs SMD of 0.28, Additional file 4: Figure S4).

\section{Narrative findings}

$\mathrm{CdM}$ use in assessing emphysema, atelectasis, hyperemia, epithelialization, and leukocyte infiltration were observed by one study. The results were not included in the statistical analysis due to the nature of reported outcomes. However, Ahmadi et al. found a significant decrease in pathological changes in CdM treated but noted that MSCs produced a more efficient amelioration of these pathological changes [29]. Sutsko et al and Aslam et al reported improvement in histologic measures of alveolarization [30, 31]. Additionally, Aslam et al documented decreased muscularization of intrapulmonary arterioles after hyperoxic injury. Studies completed by Ionescu and Wakayama similarly suggest improvements in lung fibrosis and septal thickening [32, 33]. These histological findings support the idea of conditioned media's pulmonary restorative nature.

\section{Meta-regression analysis}

Meta-regression was performed to simultaneously survey the impact of all variables on study effect. For immune cell assessment, animal species and strain, gender, age at lung injury induction, MSC origin, and timing of intervention (6 of 11 themes) were significant sources of heterogeneity $(p<0.05)$ (Table 3$)$. As for the cytokine assay assessment, gender was the only significant source for heterogeneity $(p<0.05)$ (Table 4$)$.

\section{Publication bias}

Funnel plots were created to examine the effect of study qualities and heterogeneity on publication bias (Fig. 6a \& b). Asymmetry was detected in funnel plots of immune cells and cytokines, indicating the presence of publication bias in these studies. Egger's tests were performed to formally detect statistical asymmetry, with a null hypothesis denying the existence of small study effects. The $p$ value was $<0.05$ for all tests, indicating strong evidence to reject the null hypothesis in favor of the alternative (i.e. small study effect does exist).

\section{Discussion}

The goal of systematic reviews and meta-analyses of preclinical studies is to provide a cumulative evaluation of treatments across multiple studies and to identify areas for improvement in research [26, 34, 35]. These efforts are intended to guide future studies and increase the translatability into the clinical setting. In preclinical 


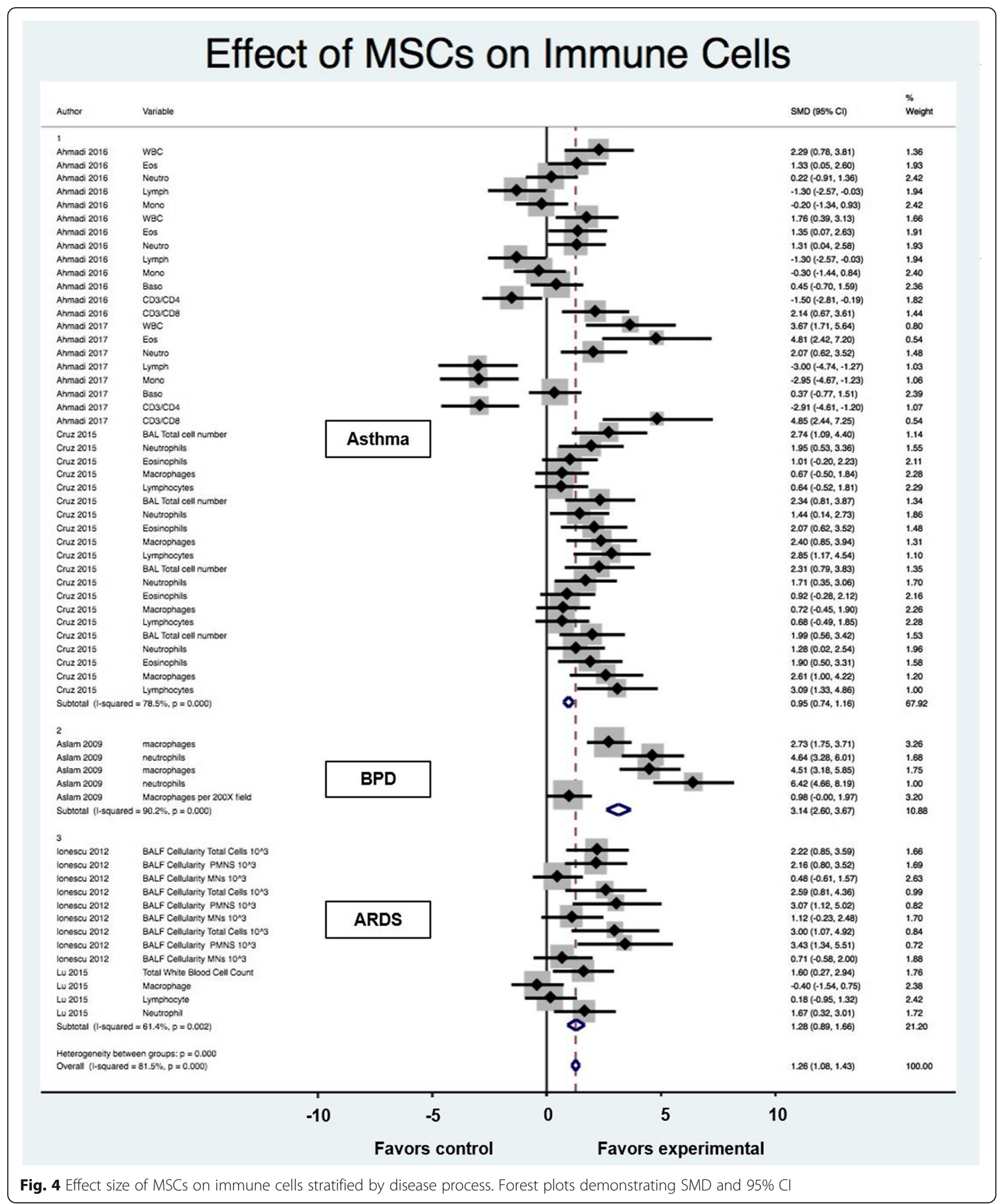

settings, MSCs improve survival, pulmonary function, and protect against structural changes via secretion of paracrine factors. Thus, in theory, MSC-derived CdM may have similar reparative, regenerative, and restorative properties as the cells themselves. However, to date there has been no systematic analysis of $\mathrm{CdM}$ as a potential therapeutic option for inflammation in animal studies modeling lung disease. This is the first preclinical 


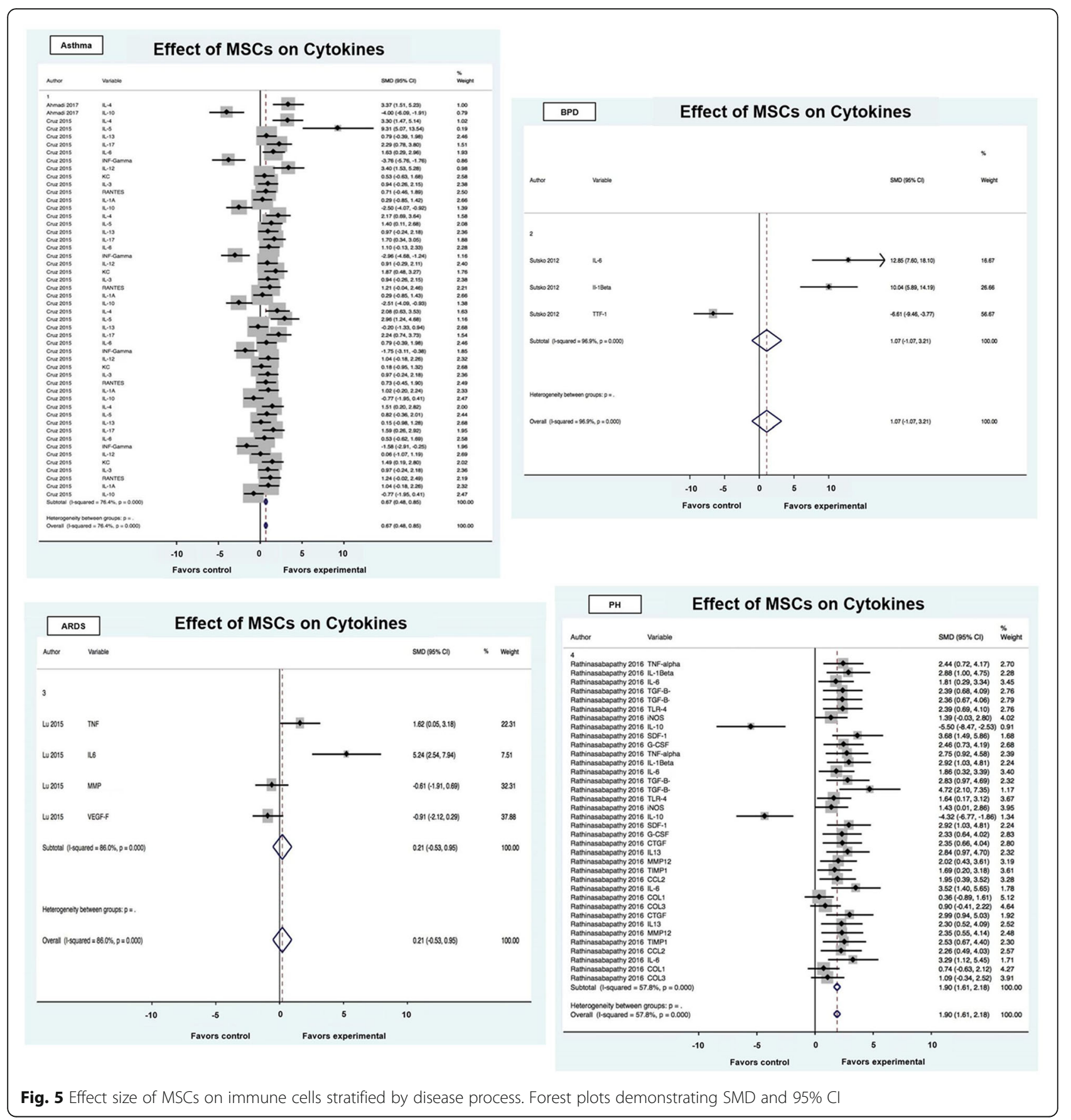

meta-analysis indicating that $\mathrm{CdM}$ has therapeutic potential in not only attenuating inflammation in animal models of pediatric/adult lung disease but are oftentimes as effective as MSCs.

In line with our CdM effects on immune cells, Augustine and colleagues also demonstrated a decrease in alveolar macrophage number after treatment of $\mathrm{BPD}$ animals with MSCs (1.90 SMD for MSCs vs. 2.42 SMD for $\mathrm{CdM}$ ) [36]. The mixed results on neutrophil and white cell counts were also observed in this meta- analysis. Similarly, a downregulation of many of the cytokines seen in our review was observed by Augustine's group. For example, IL-6 in their study had an SMD of 2.28 , while ours had an SMD of 2.23, favoring treatment with regenerative cells. A decrease in the proinflammatory cytokine IL-6 was also appreciated in a study examining $\mathrm{CdM}$ as a therapy for renal fibrosis and in a pulmonary fibrosis meta-analysis conducted by Srour et al [37]. Our overall findings that CdM attenuates inflammation was also corroborated by a review 


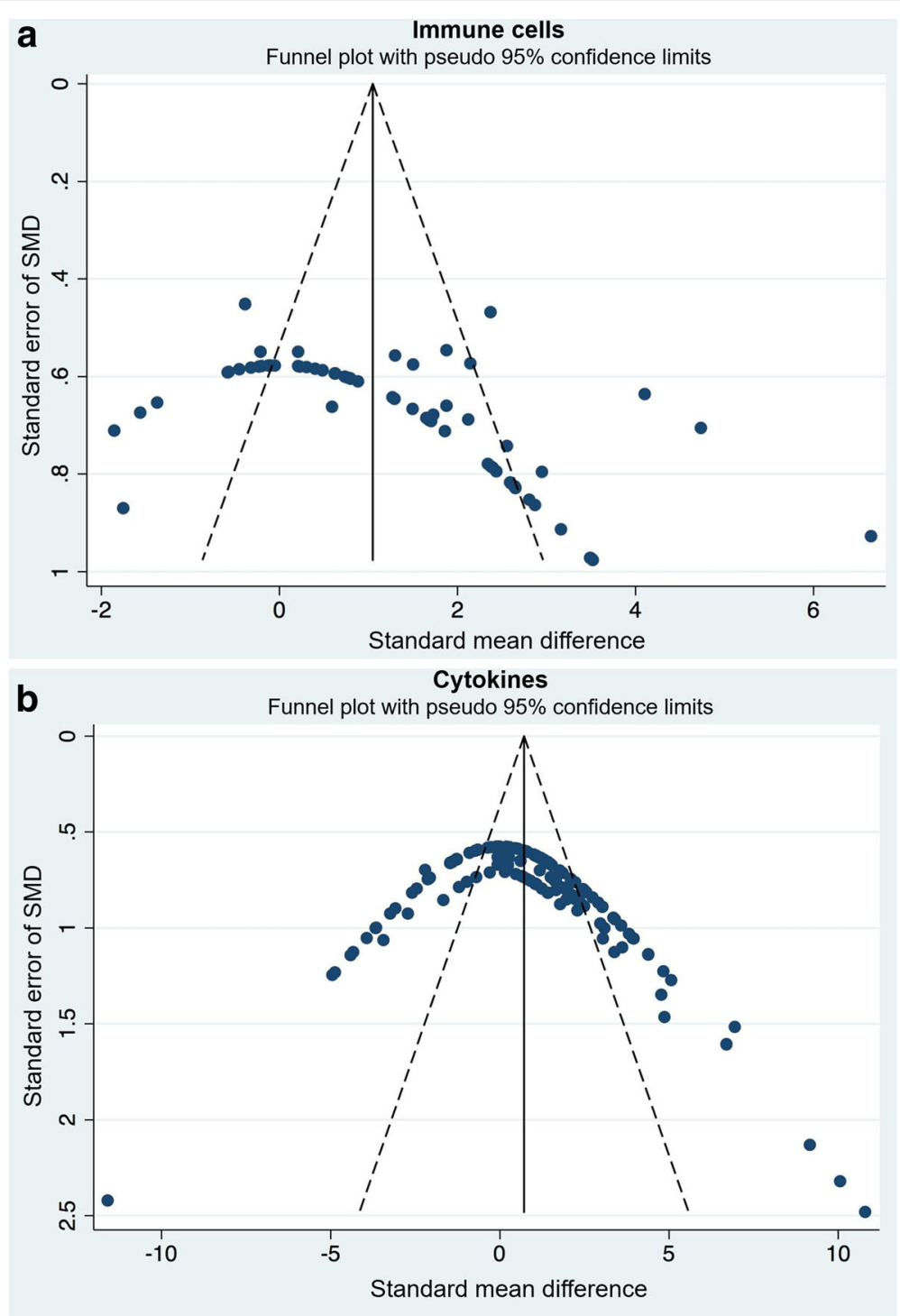

Fig. 6 Funnel plots demonstrating publication bias from included studies. Funnel plots for (a) immune cells, and (b) inflammatory cytokines

article by Mohammadipoor et al wherein they describe the anti-inflammatory effects of $\mathrm{CdM}$ in pulmonary disease [38].

Other inflammatory cytokines that were decreased in our review included IL-1 $\beta$ and TNF $\alpha$. A study by Platas et al found a decrease in the expression of IL- $1 \beta$ from osteoarthritic cells after exposure to stromal cell conditioned media [39]. A similar reduction in IL-1 $\beta$ (3.17 SMD for MSCs vs. 3.37 SMD for CdM) and TNFa was mirrored in the preclinical BPD meta-analysis by $\mathrm{Au}-$ gustine et al. TNF $\alpha$ inhibition was also seen in a study performed in an animal model of arthritis receiving CdM [40].

Herein we conduct the first preclinical meta-analysis comparing CdM to MSC as therapies for lung disease. Finding that CdM was as effective as MSCs, in an analysis of 10 studies, is a noteworthy finding in the field. Even more impressive is the fact that both therapies had the largest effect sizes on immune cells and cytokines for the same diseases (BPD and PH, respectively). Our results open opportunities for the development of therapies that may not require the cells. Clinically speaking, this suggests the potential preparation of a cell-free "drug" that can be produced, tested, and stored prior to use.

Our meta-analysis shows the need for further study to determine the ideal dosage and type of MSC-derived $\mathrm{CdM}$. While a dosage of $200 \mu \mathrm{l}$ were favored in the immune response, $100 \mu \mathrm{l}$ dosages were favored in evaluation of cytokines. Similarly, there were discrepancies in the most effective tissue source of CdM. In immune cell evaluation, bone marrow had the best outcome whereas 
adipose performed better for cytokine evaluation. These mixed results need to be further investigated in future studies to determine optimal $\mathrm{CdM}$ treatment for lung disease. Consequently, our meta-analysis should be used to guide future preclinical studies in efforts to move these findings into a clinical setting.

\section{Clinical relevance}

As of September 2nd, 2019, a search of the four lung diseases and stem cells in clinicaltrials.gov yielded 33 studies (Additional file 9: Table S5). While a similar search was undertaken with $\mathrm{CdM}$, no results were retrieved. More than half of the cell-based studies registered were targeting BPD followed by eight intervention studies in ARDS. Aside from BPD, the population of interest in the clinical trials includes adults.

Source of the stromal cells incorporated adipose, bone, umbilical cord, and menstrual tissue. The majority of studies (88\%) will use/used allogeneic stem cell transplants with a dose ranging from $500 \mathrm{k}$ cells $/ \mathrm{kg}$ of body weight to a total of 160 million cells. Of the eight completed studies, six described their early phase findings.

1. ARDS-no treatment-related adverse events and cells may improve markers of systemic injury/

inflammation

2. PH-no severe adverse events in one study, while the other study had a death of one at discharge that the safety monitoring board stated may have possibly been related to cell therapy; both studies with improvement in walking distance

3. BPD-no serious adverse events in studies with some preliminary results pointing towards a reduction in some markers of lung inflammation, one trial did not show a difference in the development of severe $\mathrm{BPD}$, yet one trial suggested a decrease in home oxygen use and improved developmental parameters

Yet some of these clinical findings appear promising, we must bear in mind that these are early phase studies and conducted in a small number of patients. Although none of the studies incorporated the use of CdM one study in particular (NCT03857841) plans to utilize bone marrow-derived MSC extracellular vesicles. These secreted particles are commonly found in $\mathrm{CdM}$ and studies as such may pave the road to future clinical trials utilizing regenerative cell-free therapies. Potential advantages in using CdM over MSCs are summarized in Additional file 10: Table S6. After further investigation, we did find one study that is examining the therapeutic potential of umbilical cord blood-derived stem cell conditioned media for alopecia (NCT03676400).

\section{Translational implications}

In spite of positive effects of regenerative cells for lung disease in animal studies many foundational questions remain. Our systematic review was hindered by the small number of studies and the large heterogeneity regarding CdM source, dose, and animal models of lung disease. Unfortunately, our review was not able to provide clear directions or recommendations for future clinical trials. However, generalizations can be extrapolated and include the following: i) CdM and MSCs improve inflammation in rodent models of pediatric/adultlung disease, ii) CdM and MSCs may be equally efficacious, iii) the intravenous route was superior in reducing inflammation compared to the intratracheal route, iv) administration of CdM after $72 \mathrm{~h}$ of the initial pulmonary insult may be preferred, and v) the source, volume, concentration, and frequency of CdM therapy is unclear.

Future translational studies should reduce bias and attempt to standardize many of the characteristics (source, dose, frequency, measures, etc) that may impact the efficacy of regenerative cell-based/derived products. A novel approach undertaking some of these knowledge gaps can be observed in cardiovascular research. The Transnational AllianCe for regenerative Therapies In Cardiovascular Syndromes (TACTICS) group has called for a paradigm shift in preclinical research to facilitate successful translation into the clinics. They recommend scientific collaborative efforts that includes sharing of protocols, knowledge, and multicenter animal studies complemented with confirmatory studies in large animals [41].

There are several limitations to our systematic review and meta-analysis. First, we had a limited number of studies and experiments. Second, we incorporated multiple animal models of pediatric/adult lung disease which most likely contributed to the high heterogeneity. Our interest was to examine the effects of regenerative therapies in animal models of lung disease; however, many of the studies used were exclusively performed in adult animals. Thus, our review did not include the wide spectrum of respiratory disease (e.g., chronic obstructive pulmonary diseases, influenza, tuberculosis, and lung cancer). This discrepancy may impede the translation of our findings. Third, none of the studies had a low risk of bias. Next, implications from this work must be viewed with caution given that the nature of the studies were all completed in small animal models of pediatric/adult lung disease. The final major barrier to our study was the current lack of systemic review or meta-analysis using CdM on models of lung disease. While the field is saturated with information on the use of MSC on lung disease, there was much less evidence on the effects of $\mathrm{CdM}$, which inhibited our ability to corroborate/refute our findings. 


\section{Conclusion}

In sum, this systematic review and meta-analysis is the first to summarize and quantify the effects of CdM as a therapeutic agent to ameliorate lung and systemic inflammation. CdM improved inflammation and was as effective as MSCs. While these findings are encouraging, we must acknowledge that the studies were performed in small animals and oftentimes positive results in rodents do not translate to the patient bedside. Studies included in this review had high heterogeneity and an overall unclear risk of bias. Further analysis must be done to move the field forward, such as determining the ideal site of CdM delivery, dosage, and timing of treatment according to disease.

\section{Supplementary information}

Supplementary information accompanies this paper at https://doi.org/10. 1186/s12931-019-1212-x.

Additional file 1: Figure S1. Effect size of CdM vs. MSC on immune cells stratified by disease process. Forest plots demonstrating SMD and 95\% Cl.

Additional file 2: Figure S2. Effect size of CdM vs. MSC on inflammatory cytokines stratified by disease process. Forest plots demonstrating SMD and $95 \% \mathrm{Cl}$.

Additional file 3: Figure S3. Effect size of CdM tissue source on immune cells in ARDS. Forest plots demonstrating SMD and $95 \% \mathrm{Cl}$.

Additional file 4: Figure S4. Effect size of CdM tissue source on inflammatory cytokines in ARDS. Forest plots demonstrating SMD and 95\% Cl.

Additional file 5: Table S1. SYRCLE 2014 protocol format.

Additional file 6: Table S2. Literature search terms.

Additional file 7: Table S3. Number of animals in studies.

Additional file 8: Table S4. CdM characteristics.

Additional file 9: Table S5. Current clinical trials in asthma, BPD, ARDS, $\mathrm{PH}$.

Additional file 10: Table S6. Advantages of CdM vs. MSCs.

\section{Abbreviations}

ARDS: Acute Respiratory Distress Syndrome; ARDS: Acute respiratory distress syndrome; BPD: Bronchopulmonary Dysplasia; BPD: Bronchopulmonary dysplasia; CAMARADES: Collaborative Approach to Meta-Analysis and Review of Data from Experimental Studies; CdM: Conditioned media; CF: Cystic fibrosis; Cl: Confidence interval; CINAHL: Cumulative Index to Nursing and Allied Health Literature; IL-1 $\beta$ : Interleukin-1 beta; IP: Intraperitoneal; IT: Intratracheal; IV: Intravenous; LPS: Lipopolysaccharide; MCT: Monocrotaline; MSCs: Mesenchymal stem/stromal cells; OVA: Ovalbumin; PH: Pulmonary Hypertension; PH: Pulmonary hypertension; PN: Postnatal; RV: Right Ventricle; SMD: Standardized mean difference; SYRCLE: Systematic Review Centre for Laboratory Animal Experimentation; TNFa: Tumor necrosis factor alpha

\section{Acknowledgements}

None.

\section{Authors' contributions}

CE and ED: study design, database search, collection and/or assembly of data, analysis of data, and first draft. RN: analysis of data and manuscript writing. AC: collection and/or assembly of data. JM: collection and/or assembly of data, manuscript writing. Axel M: manuscript writing, interpretation of data. Alvaro M: study design, database search, collection and/or assembly of data, analysis of data manuscript writing, oversight of study. All authors read and approved the final manuscript.

\section{Funding}

Supported by the National Institutes of Health NCATS KL2 TR001118, UT Health San Antonio School of Medicine pilot grant, and the Parker B. Francis Foundation (provided to Alvaro Moreira).

\section{Availability of data and materials}

The datasets generated and/or analyzed during the current study are available upon reasonable request.

\section{Competing interests}

The authors declare that they have no competing interests.

\section{Author details}

${ }^{1}$ Department of Pediatrics, Division of Neonatology, University of Texas Health-San Antonio, San Antonio, Texas 78229-3900, USA. ²Department of Pediatrics, Texas Children's Hospital, Houston, Texas, USA.

Received: 23 July 2019 Accepted: 10 October 2019 Published online: 30 October 2019

\section{References}

1. WHO | Causes of child mortality. WHO [Internet]. 2019 [cited 2019 Jul 20]; Available from: https://www.who.int/gho/child_health/mortality/causes/en/

2. Ferkol T, Schraufnagel D. The Global Burden of Respiratory Disease. Ann Am Thorac Soc [Internet]. 2014 [cited 2019 Sep 30];11(3):404-6. Available from: http://www.atsjournals.org/doi/abs/10.1513/AnnalsATS.201311-405PS

3. GBD 2015 Chronic Respiratory Disease Collaborators JB, Abajobir AA, Abate $\mathrm{KH}$, Abera SF, Agrawal A, Ahmed MB, et al. Global, regional, and national deaths, prevalence, disability-adjusted life years, and years lived with disability for chronic obstructive pulmonary disease and asthma, 1990-2015: a systematic analysis for the Global Burden of Disease Study 2015. Lancet Respir Med [Internet]. 2017 [cited 2019 Sep 30];5(9):691-706. Available from: http://www.ncbi.nlm.nih.gov/pubmed/28822787.

4. Burki TK. The economic cost of respiratory disease in the UK. Lancet Respir Med [Internet]. 2017 [cited 2019 Sep 30];5(5):381. Available from: https:// www.thelancet.com/journals/lanres/article/PIIS2213-2600(17)30108-X/fulltext

5. Bush A, Tiddens H, Silverman M. Clinical Implications of Inflammation in Young Children. Am J Respir Crit Care Med [Internet]. 2000 [cited 2019 Jul 20];162(supplement_1):S11-4. Available from: http://www.atsjournals.org/ doi/abs/10.1164/ajrccm.162.supplement_1.maic-3

6. Kumar R, Graham B. How does inflammation contribute to pulmonary hypertension? Eur Respir J [Internet]. 2018 [cited 2019 Jul 20];51(1):1702403. Available from: http://www.ncbi.nlm.nih.gov/pubmed/29371392.

7. Spadaro S, Park M, Turrini C, Tunstall T, Thwaites R, Mauri T, et al. Biomarkers for Acute Respiratory Distress syndrome and prospects for personalised medicine. J Inflamm [Internet]. 2019 [cited 2019 Jul 20];16(1):1. Available from: https://journal-inflammation.biomedcentral.com/articles/10.1186/s1295 0-018-0202-y

8. Oudijk EJD, Lammers JWJ, Koenderman L. Systemic inflammation in chronic obstructive pulmonary disease. Eur Respir J Suppl [Internet]. 2003 [cited 2019 Jul 20];46:5s-13s. Available from: http://www.ncbi.nlm.nih.gov/ pubmed/14621102.

9. Moldoveanu B, Otmishi P, Jani P, Walker J, Sarmiento X, Guardiola J, et al. Inflammatory mechanisms in the lung. J Inflamm Res 2009 [cited 2019 Jul 20];2:1-11. Available from: http://www.ncbi.nlm.nih.gov/pubmed/22096348.

10. Barnes PJ, Burney PGJ, Silverman EK, Celli BR, Vestbo J, Wedzicha JA, et al. Chronic obstructive pulmonary disease. Nat Rev Dis Prim [Internet]. 20153 [cited 2019 Jul 20];1(1):15076. Available from: http://www.nature.com/ articles/nrdp201576

11. Suter PM. Lung Inflammation in ARDS — Friend or Foe? N Engl J Med [Internet]. 2006 [cited 2019 Jul 20];354(16):1739-42. Available from: http:// www.nejm.org/doi/abs/10.1056/NEJMe068033

12. Matthay MA, Ware LB, Zimmerman GA. The acute respiratory distress syndrome. J Clin Invest. 2012 [cited 2019 Jul 20];122(8):2731-40. Available from: http://www.ncbi.nlm.nih.gov/pubmed/22850883.

13. Singer NG, Caplan Al. Mesenchymal Stem Cells: Mechanisms of Inflammation. Annu Rev Pathol Mech Dis. 2011 [cited 2019 Jul 20];6(1):45778. Available from: http://www.annualreviews.org/doi/10.1146/annurevpathol-011110-130230

14. Regulski MJ. Mesenchymal Stem Cells: \&quot;Guardians of Inflammation\&quot;. Wounds a Compend Clin Res Pract [Internet]. 2017 
[cited 2019 Jul 20];29(1):20-7. Available from: http://www.ncbi.nlm.nih.gov/ pubmed/28157686.

15. Iyer SS, Rojas M. Anti-inflammatory effects of mesenchymal stem cells: novel concept for future therapies. Expert Opin Biol Ther. 2008 [cited 2018 Jul 1]; 8(5):569-81. Available from: http://www.ncbi.nlm.nih.gov/pubmed/184 07762.

16. Horie S, Laffey JG. Recent insights: mesenchymal stromal/stem cell therapy for acute respiratory distress syndrome. F1000Research [Internet]. 2016 [cited 2016 Oct 18];5. Available from: http://www.ncbi.nlm.nih.gov/ pubmed/27408702.

17. Pedrazza L, Cunha AA, Luft C, Nunes NK, Schimitz F, Gassen RB, et al. Mesenchymal stem cells improves survival in LPS-induced acute lung injury acting through inhibition of NETs formation. J Cell Physiol [Internet]. 2017 [cited 2019 Jul 20];232(12):3552-64. Available from: http://www.ncbi.nlm.nih. gov/pubmed/28112391.

18. Danieli P, Malpasso G, Ciuffreda MC, Cervio E, Calvillo L, Copes F, et al. Conditioned medium from human amniotic mesenchymal stromal cells limits infarct size and enhances angiogenesis. Stem Cells Transl Med [Internet]. 2015 [cited 2019 Jul 20];4(5):448-58. Available from: http://www. ncbi.nlm.nih.gov/pubmed/25824141.

19. Gnecchi M, Zhang Z, Ni A, Dzau VJ. Paracrine mechanisms in adult Stem Cell signaling and therapy. Circ Res. 2008;103(11):1204-19.

20. Liang X, Ding Y, Zhang Y, Tse H-F, Lian Q. Paracrine Mechanisms of Mesenchymal Stem Cell-Based Therapy: Current Status and Perspectives. Cell Transplant [Internet]. 2014 [cited 2019 Jul 20];23(9):1045-59. Available from: http://www.ncbi.nlm.nih.gov/pubmed/23676629.

21. Vizoso FJ, Eiro N, Cid S, Schneider J, Perez-Fernandez R. Mesenchymal Stem Cell Secretome: Toward Cell-Free Therapeutic Strategies in Regenerative Medicine. Int J Mol Sci [Internet]. 2017 [cited 2017 Dec 16];18(9). Available from: http://www.ncbi.nlm.nih.gov/pubmed/28841158.

22. Moreira A, Alayli Y, Balgi S, Winter C, Kahlenberg S, Mustafa S, et al. Upcycling umbilical cords: bridging regenerative medicine with neonatology. J Matern Neonatal Med [Internet]. 2017 [cited 2017 Nov 30];110. Available from: http://www.ncbi.nlm.nih.gov/pubmed/29132234.

23. Bonfield TL, Koloze M, Lennon DP, Zuchowski B, Yang SE, Caplan Al. Human mesenchymal stem cells suppress chronic airway inflammation in the murine ovalbumin asthma model. Am J Physiol Cell Mol Physiol [Internet]. 2010 [cited 2019 Jul 20];299(6):L760-70. Available from: http://www.ncbi. nlm.nih.gov/pubmed/20817776.

24. Aslam M, Baveja R, Liang OD, Fernandez-gonzalez A, Lee C, Mitsialis SA, et al. Bone Marrow Stromal Cells Attenuate Lung Injury in a Murine Model of Neonatal Chronic Lung Disease. Am J Respir Crit Care Med. 2009;180: 1122-30.

25. Pawitan JA. Prospect of Stem Cell Conditioned Medium in 2014;2014:7-9.

26. de Vries RBM, Hooijmans CR, Langendam MW, van Luijk J, Leenaars M, Ritskes-Hoitinga $\mathrm{M}$, et al. A protocol format for the preparation, registration and publication of systematic reviews of animal intervention studies. Evidence-based Preclin Med [Internet]. 2015 Aug [cited 2016 Dec 2];2(1): e00007. Available from: http://doi.wiley.com/10.1002/ebm2.7

27. Mitchell C, Provost K, Niu N, Homer R, Cohn L. IFN-y acts on the airway epithelium to inhibit local and systemic pathology in allergic airway disease. J Immunol [Internet]. 2011 [cited 2019 Jul 16];187(7):3815-20. Available from: http://www.ncbi.nlm.nih.gov/pubmed/21873527.

28. Stahlman MT, Gray ME, Whitsett JA. Expression of thyroid transcription factor-1(TTF-1) in fetal and neonatal human lung. J Histochem Cytochem [Internet]. 1996 [cited 2019 Jul 20];44(7):673-8. Available from: http://www. ncbi.nlm.nih.gov/pubmed/8675988.

29. Ahmadi M, Rahbarghazi R, Aslani MR, Shahbazfar A-A, Kazemi M, Keyhanmanesh R. Bone marrow mesenchymal stem cells and their conditioned media could potentially ameliorate ovalbumin-induced asthmatic changes. Biomed Pharmacother [Internet]. 2017 [cited 2019 Jul 20]:85:28-40. Available from: http://www.ncbi.nlm.nih.gov/pubmed/27930984.

30. Sutsko RP, Young KC, Ribeiro A, Torres E, Rodriguez M, Hehre D, et al. Longterm reparative effects of mesenchymal stem cell therapy following neonatal hyperoxia-induced lung injury. Pediatr Res. 2013 [cited 2019 Jul 20];73(1):46-53. Available from: http://www.ncbi.nlm.nih.gov/pubmed/23138401.

31. Aslam M, Baveja R, Liang OD, Fernandez-Gonzalez A, Lee C, Mitsialis SA, et al. Bone marrow stromal cells attenuate lung injury in a murine model of neonatal chronic lung disease. Am J Respir Crit Care Med [Internet]. 2009 [cited 2017 Feb 9]:180(11):1122-30. Available from: http://www ncbinlm.nih. gov/pubmed/19713447.
32. Ionescu L, Byrne RN, van Haaften T, Vadivel A, Alphonse RS, Rey-Parra GJ, et al. Stem cell conditioned medium improves acute lung injury in mice: in vivo evidence for stem cell paracrine action. Am J Physiol Cell Mol Physiol [Internet]. 2012 [cited 2019 Jul 20];303(11):L967-77. Available from: http:/www.ncbi.nlm.nih.gov/pubmed/23023971.

33. Wakayama $\mathrm{H}$, Hashimoto N, Matsushita Y, Matsubara K, Yamamoto N, Hasegawa $Y$, et al. Factors secreted from dental pulp stem cells show multifaceted benefits for treating acute lung injury in mice. Cytotherapy [Internet]. 2015 [cited 2019 Jul 20];17(8):1119-29. Available from: http:// www.ncbi.nlm.nih.gov/pubmed/26031744.

34. Sandercock P, Roberts I, Vries RB de, Leenaars M, Ritskes-Hoitinga M, Langendam MW, et al. Systematic reviews of animal experiments. Lancet [Internet]. 2002 Aug [cited 2017 Jan 24];360(9333):586. Available from: http://linkinghub.elsevier.com/retrieve/pii/S0140673602098124

35. de Vries RBM, Wever KE, Avey MT, Stephens ML, Sena ES, Leenaars M. The usefulness of systematic reviews of animal experiments for the design of preclinical and clinical studies. ILAR J [Internet]. 2014 [cited 2019 Jul 20]; 55(3):427-37. Available from: http://www.ncbi.nlm.nih.gov/ pubmed/25541545.

36. Augustine S, Avey MT, Harrison B, Locke T, Ghannad M, Moher D, et al. Mesenchymal Stromal Cell Therapy in Bronchopulmonary Dysplasia: Systematic Review and Meta-Analysis of Preclinical Studies. Stem Cells Trans Med [Internet]. 2017 [cited 2018 Oct 15];6(12):2079-93. Available from: http://www.ncbi.nlm.nih.gov/pubmed/29045045

37. Srour N, Thébaud B. Mesenchymal stromal Cells in animal Bleomycin pulmonary fibrosis models: a systematic review. Stem Cells Transl Med. 2015;:1500-10

38. Mohammadipoor A, Antebi B, Batchinsky Al, Cancio LC. Therapeutic potential of products derived from mesenchymal stem/stromal cells in pulmonary disease. Respir Res [Internet]. 2018 [cited 2019 Jul 19];19(1):218. Available from: http://www.ncbi.nlm.nih.gov/pubmed/30413158.

39. Platas J, Guillén MI, del Caz MDP, Gomar F, Mirabet V, Alcaraz MJ. Conditioned media from adipose-tissue-derived mesenchymal stem cells downregulate degradative mediators induced by interleukin-1 $\beta$ in osteoarthritic chondrocytes. Mediators Inflamm [Internet]. 2013 [cited 2019 Jul 20];2013:357014. Available from: http://www.ncbi.nlm.nih.gov/ pubmed/24363499.

40. Kay AG, Long G, Tyler G, Stefan A, Broadfoot SJ, Piccinini AM, et al. Mesenchymal Stem Cell-Conditioned Medium Reduces Disease Severity and Immune Responses in Inflammatory Arthritis. Sci Rep [Internet]. 2017 [cited 2019 Jul 20];7(1):18019. Available from: http://www.ncbi.nIm.nih.gov/ pubmed/29269885.

41. Chamuleau SAJ, van der Naald M, Climent AM, Kraaijeveld AO, Wever KE, Duncker DJ, et al. Translational Research in Cardiovascular Repair. Circ Res [Internet]. 2018 [cited 2018 Oct 15];122(2):310-8. Available from: http:// www.ncbi.nlm.nih.gov/pubmed/29348252.

\section{Publisher's Note}

Springer Nature remains neutral with regard to jurisdictional claims in published maps and institutional affiliations.

Ready to submit your research? Choose BMC and benefit from:

- fast, convenient online submission

- thorough peer review by experienced researchers in your field

- rapid publication on acceptance

- support for research data, including large and complex data types

- gold Open Access which fosters wider collaboration and increased citations

- maximum visibility for your research: over $100 \mathrm{M}$ website views per year

At BMC, research is always in progress.

Learn more biomedcentral.com/submission 